American Journal of Infectious Diseases 4 (4): 257-261, 2008

ISSN 1553-6203

(C) 2008 Science Publications

\title{
Estimating HIV-1 Incidence Using the Serologic Testing Algorithm for Recent HIV Seroconversion at HIV Counseling and Testing Centers in the City of São Paulo, Brazil
}

\author{
${ }^{1}$ Katia Cristina Bassichetto, ${ }^{2}$ Denise Pimentel Bergamaschi, ${ }^{3}$ Maria Amelia Veras, ${ }^{4}$ Maria Cecilia Araripe \\ Sucupira, ${ }^{1}$ Fabio Mesquita and ${ }^{4}$ Ricardo Sobhie Diaz \\ ${ }^{1}$ Coordenação Municipal de DST/AIDS - Secretaria Municipal de Saúde de \\ São Paulo, São Paulo, SP, Brazil \\ ${ }^{2}$ Departamento de Epidemiologia/Faculdade de Saúde Pública - Universidade de São \\ Paulo, São Paulo, SP, Brazil \\ ${ }^{3}$ Departamento de Medicina Social da Faculdade de Ciências Médicas da Santa Casa de \\ São Paulo, São Paulo, SP, Brasil; \\ ${ }^{4}$ Retrovirology Laboratory - Paulista School of Medicine Federal University of são Paulo, \\ São Paulo, SP, Brazil
}

\begin{abstract}
The network of HIV counseling and testing centers in São Paulo, Brazil is a major source of data used to build epidemiological profiles of the client population. In this study, HIV-1 incidence from November of 2000 to April of 2001 was estimated, comparing recently infected individuals to those with long-standing infection in terms of epidemiological and socio-behavioral aspects. To identify recent infection, less sensitive ELISA was employed. The overall incidence of HIV-1 infection among the clients was 0.53/100/year (95\% CI: $0.31-0.85 / 100 /$ year): $0.77 / 100 /$ year for males (95\% CI: 0.42-1.27/100/year) and 0.22/100/year (95\% CI: 0.05-0.59/100/year) for females. Overall HIV-1 prevalence was $3.2 \%$ (95\% CI: $2.8-3.7 \%$ ), being $4.0 \%$ among males (95\% CI: $3.3-4.7 \%$ ) and $2.1 \%$ among females (95\% CI: 1.6-2.8\%). Recent infections accounted for $15 \%$ of the total (95\% CI: $10.2-20.8 \%)$. Recent infection correlated with being younger and male $(\mathrm{p}=0.019)$. Therefore, recent infection was more common among younger males and older females.
\end{abstract}

Key words: HIV-1/immunology, HIV infections/epidemiology, HIV seroprevalence, Seroepidemiologic Studies

\section{INTRODUCTION}

In December of 2001, the total number of AIDS cases reported in the city of São Paulo was 51,841, corresponding to $22 \%$ of all AIDS cases reported in Brazil as a whole (http://www.aids.gov.br). The HIV-1 epidemic in Brazil is characterized based on the number of reported AIDS cases and the number of HIV-infected pregnant women (and their infected children), as well as on HIV-1 sentinel surveillance of certain population groups. In several studies, recent HIV-1 infection has been described using a less sensitive ELISA ${ }^{[1-4]}$. This detection strategy, known as the serologic testing algorithm for recent HIV seroconversion (STARHS) ${ }^{[3]}$, has been used to estimate HIV-1 incidence and to investigate epidemiological aspects in newly infected individuals.

The city of São Paulo, where nearly $25 \%$ of all Brazilian AIDS cases occur, is recognized as the epicenter of the AIDS epidemic in Brazil (http://www.aids.gov.br). The São Paulo Municipal STD/HIV Network comprises 22 outpatient clinics located throughout the city. One important element of this network is the service provided by the HIV counseling and testing (HIV CT) centers, which conduct STD/HIV testing and provide prevention counseling. The health care system is also a major source of data, enabling studies of HIV-1 prevalence and epidemiological profiles in client populations.

In the STARHS methodology, serum samples testing positive for HIV-1 in the initial ELISA are

Corresponding Author: Ricardo Sobhie Diaz, Laboratório de Retrovirologia, Universidade Federal de São Paulo-EPM, R. Pedro de Toledo, 781-16 andar, São Paulo, SP-04039-032- Brazil, Tel: +55 (11) 9109-0445; fax: +55 (11) $4192-3176$ 
retested. In the second test, a less sensitive ELISA is performed. The less sensitive ELISA protocol uses higher serum dilutions and shorter incubation periods. If a sample tests positive in the conventional ELISA and negative in the less sensitive ELISA, the viral infection is presumed to have occurred within the preceding 170 days and the patient is classified as having been recently infected with HIV-1. This system allows the incidence of recent infection to be estimated.

In this study, we used the STARHS methodology to calculate the number of recent HIV-1 infections in samples collected at HIV CT centers between November of 2000 and April of 2001. We also studied the epidemiological and socio-behavioral aspects of HIV-positive individuals that had been recently infected as well as those of individuals presenting long-standing infection.

\section{MATERIALS AND METHODS}

Study design: This was a cross-sectional study using information gathered from questionnaires administered between November of 2000 and April of 2001 during the course of routine HIV CT center services provided before and after testing. A total of five HIV CT centers were involved in this study and are identified by their locations: Henfil; Lapa; Pirituba; Santo Amaro; and São Miguel.

Starhs: Patient blood samples were collected in Vacutainer tubes (Becton-Dickinson, Rutherford, NJ, USA). On the same day, samples were transferred to one of the three public reference laboratories for storage. All samples were tested twice using conventional HIV-1 ELISA. Double-negative samples were considered HIV-1 negative. Samples for which the ELISA results were inconclusive, as well as those testing positive for HIV-1 only once, were further analyzed by Western blot according to the guidelines established by the Brazilian Health Ministry ${ }^{[3]}$.

Samples classified as HIV-1 positive were sent to the Retrovirology Laboratory of the Federal University of São Paulo to be analyzed using STARHS. The Vironostika HIV-1 Micro-ELISA System kit was used (LS HIV-1 EIA; Organon Teknika, Durham, NC, USA) according to the manufacturer instructions.

Serum samples were diluted to 1:20,000, and incubation times were modified. Vironostika negative controls, Centers for Disease Control and Prevention calibrators, high-positive controls, and low-positive controls were also diluted and incubated according to the manufacturer instructions. Sample selection and control analysis were performed in triplicate. Sample optical density (SOD) was calculated using the equation:

$$
\begin{aligned}
\mathrm{SOD}= & \left(\mathrm{OD}_{\text {sample }}-\mathrm{OD}_{\text {median negative control }}\right) / \\
& \left(\mathrm{OD}_{\text {median calibrator }}-\mathrm{OD}_{\text {median negative control }}\right)
\end{aligned}
$$

Samples in which the SOD screening value was below 2.0 were tested in triplicate as a confirmatory measure. The SOD was calculated based on median OD values measured in triplicate. Samples in which the confirmed SOD value was below 1.0 were determined to have originated from patients having acquired the HIV-1 infection within the preceding 170 days $^{[3]}$.

Statistical analysis: In our analysis, the outcome variable was the timing of HIV-1 infection (recent or not). Explanatory and control variables included ethnicity, education, marital status, type of exposure and use of condoms. The annual HIV-1 incidence, expressed as the percentage of new cases/year that were identified as cases of recent infection, was calculated for the population as a whole and by gender using the estimator included in the Vironostika-LS protocol:

$$
\% \text { Per year }=\frac{\begin{array}{l}
\text { number of recently } \\
\text { inf ected patients }
\end{array}}{\begin{array}{l}
\text { Number of HIV }-1 \text { negative individuals } \\
\text { +number of recently infected patients }
\end{array}} \times \frac{365.25^{*} \times 100}{170}
$$

Study period $=182.5$ days

The prevalence of HIV-1 infection was calculated as the ratio between the number of HIV-1-positive individuals and the total number of individuals tested in the first sample. For the incidence and prevalence of recent HIV-1 infections, 95\% confidence intervals ( $95 \%$ CIs) were calculated. The $95 \%$ CIs were used not only for estimation but also to compare the prevalence and incidence by gender. Correlations between the outcome and explanatory variables were identified using the chi-square test or Fisher's exact test, together with linear trend tests. In this study, multivariable analysis was unnecessary and thus was not performed. The Epi Info, version $6.0^{[5]}$, and Stata, version 7.0 (Stata, College Station, TX, USA), programs were used to build and analyze the database.

The Ethics in Research Committee of the Training and Reference Centre for STD/AIDS (São Paulo State Health Secretariat) approved the study, and all participants gave written informed consent.

\section{RESULTS}

A total of 6000 individuals (3486 males and 2514 females) were tested, of which 194 (140 males and 54 


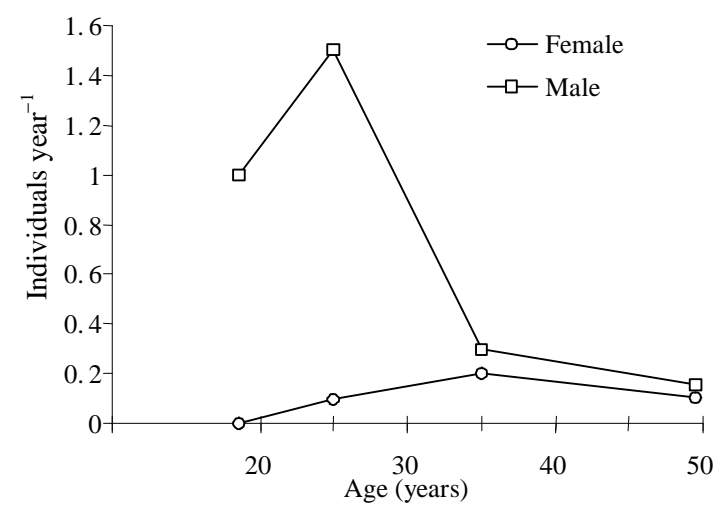

Fig. 1: Distribution of HIV-1 patients by gender and age. Data compiled for the November of 2000 to April of 2001 period from four counselling and testing centres within the São Paulo Municipal Health Network System

females) were found to be HIV-1 positive and were included in the study sample. Of those 194, 105 had been tested at Henfil, 10 at Lapa, 9 at Pirituba, 23 at Santo Amaro, and 31 at São Miguel. The overall prevalence of HIV-1 infection was 3.2\% (95\% CI: 2.8$3.7 \%$ ). Prevalence varied by gender, being $4.0 \%$ among males (95\% CI: $3.3-4.7 \%$ ) and $2.1 \%$ among females (95\% CI: 1.6-2.8\%). Among the individuals identified as HIV-1 positive, $15 \%$ were classified as having been recently infected (95\% CI: 10.2-20.8\%). The overall incidence of recent HIV-1 infection, regardless of gender, was $0.53 / 100 /$ year $\quad(95 \% \quad \mathrm{CI}: \quad 0.31-$ 0.85/100/year), being 0.77/100/year for males (95\% CI: $0.42-1.27 / 100 /$ year) and $0.22 / 100 /$ year for females (95\% CI: 0.05-0.59/100/year). Cases of recent infection tended to occur more frequently in individuals under 29 years of age $(p=0.043)$. This correlation was stronger for men under 29years of age $(p=0.019)$. Figure 1 shows that the patterns of age distribution among recently infected individuals differ by gender. The incidence among males increased significantly by 20 years of age, peaking at 25 and dropping markedly thereafter. Among women, there was a trend toward increased incidence after 25 years of age, peaking at 35 and decreasing thereafter. In Table 1, it can be seen that recent HIV-1 infection did not correlate in a statistically significant manner with ethnicity $(\mathrm{p}=0.702)$, education $(\mathrm{p}=0.555)$, marital status $(\mathrm{p}=0.178)$, employment status $(\mathrm{p}=0.810)$, income ( $\mathrm{p}=0.549)$, use of condoms $(p=0.480)$, type of exposure $(p=0.314)$, or number of partners $(\mathrm{p}=0.851)$.
Table 1: Distribution of individuals by socio-demographic and HIV1 serological status. Data compiled for the November of 2000 to April of 2001 period from four counseling and testing centers within the São Paulo Municipal Health Network System

\begin{tabular}{|c|c|c|c|c|c|}
\hline \multirow[b]{3}{*}{ Characteristic } & \multicolumn{4}{|c|}{ Recent Infection } & \multirow[b]{3}{*}{$\mathrm{p}$} \\
\hline & \multicolumn{2}{|c|}{ Yes } & \multicolumn{2}{|l|}{ No } & \\
\hline & $\mathrm{n}$ & $\%$ & $\mathrm{n}$ & $\%$ & \\
\hline Gender & & & & & \multirow{3}{*}{$0.168 *$} \\
\hline Male & 24 & 17.1 & 116 & 82.9 & \\
\hline Female & 5 & 9.3 & 49 & 90.7 & \\
\hline \multicolumn{6}{|l|}{ Age (years) } \\
\hline$<20$ & 3 & 33.3 & 6 & 66.7 & \multirow[t]{4}{*}{$0.043^{\dagger}$} \\
\hline $20-29$ & 16 & 20.8 & 61 & 79.2 & \\
\hline $30-39$ & 5 & 7.6 & 61 & 92.4 & \\
\hline$>40$ & 5 & 11.9 & 37 & 88.1 & \\
\hline \multicolumn{6}{|l|}{ Ethnicity } \\
\hline White & 16 & 15.5 & 87 & 84.5 & \multirow[t]{4}{*}{$0.702^{\dagger}$} \\
\hline Black & 4 & 21.1 & 15 & 79.0 & \\
\hline Mixed & 8 & 18.2 & 36 & 81.8 & \\
\hline Asian & 0 & - & 7 & 100.0 & \\
\hline \multicolumn{6}{|l|}{ Education } \\
\hline None & 12 & 15.6 & 65 & 84.4 & \multirow[t]{4}{*}{$0.555^{\dagger}$} \\
\hline Elementary school & 7 & 15.2 & 39 & 84.8 & \\
\hline High School & 9 & 17.7 & 42 & 82.4 & \\
\hline University degree & 0 & - & 12 & 100.0 & \\
\hline \multicolumn{6}{|l|}{ Marital Status } \\
\hline Single & 21 & 18.9 & 90 & 81.1 & \multirow[t]{2}{*}{$0.178^{*}$} \\
\hline Married & 7 & 11.1 & 56 & 88.9 & \\
\hline \multicolumn{6}{|l|}{ Employment status } \\
\hline Employed & 22 & 14.6 & 129 & 85.4 & \multirow[t]{2}{*}{$0.810^{*}$} \\
\hline Unemployed & 7 & 16.3 & 36 & 83.7 & \\
\hline \multicolumn{6}{|l|}{ Income } \\
\hline 0 & 3 & 11.5 & 31 & 20.7 & \multirow[t]{4}{*}{$0.549^{\dagger}$} \\
\hline $1-2$ & 3 & 11.5 & 27 & 18.0 & \\
\hline $3-4$ & 13 & 50.0 & 62 & 41.3 & \\
\hline $5-6$ & 7 & 26.9 & 30 & 20.0 & \\
\hline \multicolumn{6}{|l|}{ Exposure category } \\
\hline Heterosexual & 11 & 12.5 & 77 & 87.5 & \multirow[t]{2}{*}{$0.314 *$} \\
\hline Homosexual & 10 & 22.2 & 35 & 77.8 & \\
\hline Bisexual & 7 & 18.0 & 32 & 82.1 & \\
\hline \multicolumn{6}{|l|}{ Use of condoms } \\
\hline Always & 6 & 11.5 & 46 & 88.5 & \multirow[t]{3}{*}{$0.480^{\dagger}$} \\
\hline Sporadic & 17 & 19.5 & 70 & 80.5 & \\
\hline Never & 5 & 14.7 & 29 & 85.3 & \\
\hline \multicolumn{6}{|l|}{ Number of partners } \\
\hline Single & 8 & 17.0 & 39 & 83.0 & \multirow[t]{2}{*}{$0.851^{*}$} \\
\hline Multiple & 19 & 15.8 & 101 & 84.2 & \\
\hline
\end{tabular}

*Pearson's chi-square test

'Fisher's exact test

Expressed as the number of times the local minimum wage

\section{DISCUSSION}

Our study addressed the incidence of HIV-1 among individuals seeking counseling or treatment at the HIV CT centers of the STD/AIDS network in the city of São Paulo. Rates of recent infection were estimated using the STARHS methodology, which has been successfully employed for this purpose in various other studies $^{[2,6-9]}$. 
The HIV-1 prevalence observed in the present study $(3.2 \%)$ is lower than the $4.4 \%$ reported among HIV CT centre users in a previous study conducted in the city of São Paulo ${ }^{[10]}$ and the $7.1 \%$ found among such clients in the city of Santos, also located in the state of São Paulo.(11) However, it is higher than the $2.1 \%$ observed in the city of San Francisco, California $(\mathrm{USA})^{[2]}$.

Similar numbers of men and women were tested at the HIV CT centers. However, HIV-1 infections were more prevalent among men, likely reflecting historical aspects of the local epidemic in terms of demographics and exposure. Alves et al. found the same to be true for HIV CT centre users in the city of Santos ${ }^{[11]}$. The results of the present study indicate that the incidence of recent HIV-1 infection is comparable between men and women, which is also in agreement with the findings of Alves et al. ${ }^{[11]}$ In view of the trend toward a decrease in the male-to-female ratio of AIDS cases in Brazil, which dropped from 23:1 in the mid-1980s to 1.5:1 in 2005 (www.prefeitura.sp.gov.br/dstaids), these findings were expected.

The incidence of recent HIV-1 infection observed in the present study $(0.53 / 100 /$ year $)$ was lower than that described in previous studies involving diverse population groups (men who have sex with men, injection drug users, and others) and analysis of stored serum samples. Studies conducted in Brazil have demonstrated incidence rates varying from 1.2 to 2.8/100/year ${ }^{[10,11]}$.

\section{CONCLUSION}

In the present study, $60 \%$ of the individuals tested were men who have sex with men. Nevertheless, we found no statistically significant difference between men who have sex with men and heterosexual men in terms of recent infection $(\mathrm{p}=0.314)$. This might indicate either similarity between the two exposure groups or low statistical power to detect differences (related to the small sample size). A sound estimate of HIV-1 incidence and the epidemiological aspects of newly infected populations will only be made when larger numbers of recently infected individuals are identified using STARHS. It is of note that, in the present study, a statistically significant correlation between recent infection and age was observed only for males that were homosexual or bisexual $(\mathrm{p}=0.003)$. This group seems to be at greater risk of acquiring HIV1 infection in this area in Brazil, and similar findings have been reported for young gay men in other areas of the world ${ }^{[12]}$. However, among women living in this region, the risk for recent $\mathrm{HIV}-1$ infection seems to peak at a later age, as shown in Fig. 1. Further prospective studies should be conducted in order to confirm these differences and seek to explain them. In addition, molecular epidemiological studies characterizing cases of recent HIV-1 infection will certainly lead to a better understanding of HIV-1 epidemics and their trends in terms of the profile of the virus and primary resistance to antiretroviral drugs. We recognize the fact that the retrospective nature of this study, as well as the small number of individuals analyzed, might have limited our evaluation of epidemiological aspects. However, as previously mentioned, preliminary analyses generated here can provide insights for the design of future studies.

Identifying cases of recent HIV-1 infection has clinical implications, allowing the implementation of early intervention measures, which can result in better control of virus replication and propagation during primary infection. This strategy might also help define the inclusion criteria for therapeutic and pathogenesis studies. In addition, since it also identifies populations at greater risk for HIV-1 infection, profiling the recently infected could be considered a prevention tactic. Furthermore, the inclusion of HIV-1 seronegative individuals in studies focusing on recent infections may help us understand new components of this epidemic and thereby lead to the development of better intervention measures.

\section{ACKNOWLEDGEMENT}

This research was funded by a research grant from the Fundação de Amparo à Pesquisa do Estado de São Paulo (FAPESP, Foundation for the Support of Research in the State of São Paulo; grant no. 98/14381-4).

\section{REFERENCES}

1. Janssen, R.S., G.A. Satten and S.L. Stramer et al., 1998. New testing strategy to detect early HIV-1 infection for use in incidence estimates and for clinical and prevention purposes. Jama, 280: 42-48. http://jama.amaassn.org/cgi/content/abstract/280/1/42.

2. McFarland, W., M.P. Busch and T.A. Kellogg et al., 1999. Detection of early HIV infection and estimation of incidence using a sensitive/lesssensitive enzyme immunoassay testing strategy at anonymous counseling and testing sites in San Francisco. J. Acquir Immune Defic. Syndr., 22: 484-489.

http://www.ncbi.nlm.nih.gov/pubmed/10961610. 
3. Kothe, D., R.H. Byers and S.P. Caudill et al., 2003. Performance characteristics of a new less sensitive HIV-1 enzyme immunoassay for use in estimating HIV seroincidence. J. Acquir Immune Defic. Syndr., 33: 625-634. http://www.ncbi.nlm.nih.gov/pubmed/12902808.

4. Rawal, B.D., A. Degula and L. Lebedeva et al., 2003. Development of a new less-sensitive enzyme immunoassay for detection of early HIV-1 infection. J. Acquir Immune Defic. Syndr., 33: 349-355. http://www.ncbi.nlm.nih.gov/pubmed/12843746.

5. Dean, A.G., T.G. Arner and S. Sangam et al., 2002. Epi Info, a database and statistics program for public health professionals for use on Windows 95, 98, ME, NT, 2000 and XP computers. In. 6.0 end. Vol. 32, Series Epi Info, a database and statistics program for public health professionals for use on Windows 95, 98, ME, NT, 2000 and XP computers. Atlanta: Centers for Disease Control and Prevention.

6. Kellogg, T.A., L. Loeb and J. Dilley et al., 2005. Comparison of Three Methods to Measure HIV Incidence Among Persons Seeking Voluntary, Anonymous Counseling and Testing. J. Acquir Immune Defic. Syndr., 39: 112-120. http://cat.inist.fr/?aModele $=$ afficheN\&cpsidt $=1675$ 6629.

7. McFarland, W., T.A. Kellogg and J. Dilley et al., 1997. Estimation of human immunodeficiency virus (HIV) seroincidence among repeat anonymous testers in San Francisco. Am. J. Epidemiol., 146: 662-664.

http://aje.oxfordjournals.org/cgi/content/abstract/14 6/8/662.
8. McFarland, W., T.A. Kellogg and B. Louie et al., 2000. Low estimates of HIV seroconversions among clients of a drug treatment clinic in San Francisco, 1995 to 1998. J. Acquir Immune Defic. Syndr., 23: 426-429. http://www.ncbi.nlm.nih.gov/pubmed/10866236.

9. Turchi, M.D., R.S. Diaz and C.M. Martelli et al, 2002. Genetic diversity and HIV-1 incidence estimation among cocaine users in Sao Paulo, Brazil. J. Acquir Immune Defic. Syndr., 30: 527532.

http://cat.inist.fr/?aModele=afficheN\&cpsidt=1384 5960.

10. Bassichetto, K.C., F. Mesquita and C. Zacaro et al., 2004. Perfil epidemiológico dos usuários de um Centro de Testagem e Aconselhamento para DST/HIV da Rede Municipal de São Paulo, com sorologia positiva para o HIV. Rev. Bras. Epidemiol., 7: 302-310.

http://biblioteca.universia.net/ficha.do?id=1109082

11. Alves, K., K.P. Shafer and M. Caseiro et al., 2003. Risk factors for incident HIV infection among anonymous HIV testing site clients in Santos, Brazil: 1996-1999. J. Acquir Immune Defic. Syndr., 32: 551-559. http://cat.inist.fr/?aModele $=$ afficheN\&cpsidt $=1472$ 0967.

12. Weber, A.E., K.J. Craib and K. Chan et al., 2003. Determinants of HIV serconversion in an era of increasing HIV infection among young gay and bisexual men. Aids, 17: 774-777. http://cat.inist.fr/?aModele $=$ afficheN\&cpsidt $=1480$ 4157. 